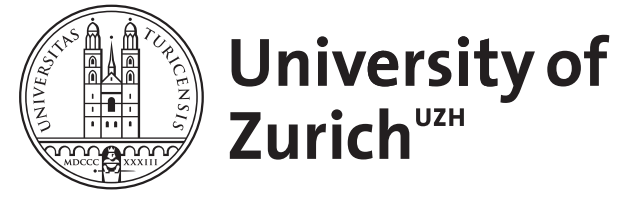

Zurich Open Repository and Archive

University of Zurich

University Library

Strickhofstrasse 39

CH-8057 Zurich

www.zora.uzh.ch

Year: 2014

\title{
Studentenprojekt Arosa - Eindrücke eines Teilnehmers und eines Tutors
}

Badertscher, N ; Senn, O

Posted at the Zurich Open Repository and Archive, University of Zurich ZORA URL: https://doi.org/10.5167/uzh-96696

Journal Article

Published Version

Originally published at:

Badertscher, N; Senn, O (2014). Studentenprojekt Arosa - Eindrücke eines Teilnehmers und eines Tutors. PrimaryCare, 14(10):175. 


\section{Studentenprojekt Arosa - Eindrücke eines Teilnehmers und eines Tutors}

\section{Der Student: Interview mit David Schilter}

\section{Möchtest Du Dich kurz vorstellen?}

Ich bin David Schilter und habe im Herbst 2013 an der Universität Zürich mein Staatsexamen gemacht. Aktuell arbeite ich an meiner Dissertation im Horten-Zentrum Zürich. Im April 2014 werde ich im Spital Altdorf als Assistenzarzt auf der Chirurgie beginnen und danach voraussichtlich auf die Innere Medizin wechseln. Meine Zukunft sehe ich in der allgemeinen Inneren Medizin, ich bin jedoch noch nicht sicher, ob ambulant oder stationär.

Was hat Dich motiviert, Dich anzumelden?

Ich hatte per Zufall im März einen freien Monat im Wahlstudienjahr und somit gut Zeit. Ich fand, die Beschreibung klinge interessant, zudem ist eine Kongressteilnehme sonst als Student nicht so ohne weiteres möglich. Ich konnte dann auch noch einen Studienkollegen motivieren, mitzukommen. Es wäre eine gewisse Barriere für mich gewesen, wenn ich gar keinen anderen Teilnehmer gekannt hätte. Ein wichtiger Motivationsgrund war ehrlich gesagt auch Arosa als Ort, ich freute mich auf ein Wochenende in den Bergen.

Was waren Deine Erwartungen an das Studentenprojekt?

Ich hatte keine konkreten Erwartungen an das Projekt, sondern war einfach neugierig, wie so ein Hausarztkongress abläuft. Es war mir bis zu diesem Zeitpunkt übrigens nicht bewusst, dass es überhaupt spezifisch hausärztliche Kongresse gibt.

Wie hast Du die Teilnahme am Kongress (Plenum, Pausen im Kongresszentrum) erlebt?

Die älteren Hausärzte freuten sich sehr darüber, dass wir «Jungen» an ihren Kongress kamen, und sie waren sehr hilfsbereit und interessiert. Besonders motivierend fand ich jedoch den Kontakt zu den anwesenden jüngeren Hausärzten, sie waren doch noch etwas näher an uns Studenten und unserer Situation dran als die ältere Generation. Dort war vielleicht der grosse Altersunterschied eine gewisse Barriere für einen ungezwungenen Austausch.

Wie hast Du die Teilnahme am spezifischen Studentenprogramm (Workshop, Rahmenprogramm) erlebt?

Der Workshop am Freitagmorgen war mit dem Thema «Thoraxschmerz» zwar nicht wirklich neu für uns, es ist mir jedoch in Erinnerung geblieben, dass der Patient eben kein Herzproblem hatte, sondern «nur» unglücklich verliebt war. Da der Hausarzt den Patienten und seine Vorgeschichte gut kannte, konnte er die Beschwerden richtig einordnen. Dieser deutliche Gegensatz zum Ablauf und dem Vorgehen im Spital war eindrücklich für mich.

Das gemeinsame Abendessen am Donnerstag war vor allem für den Austausch zwischen den Studenten und den Tutoren wertvoll sowie als «Eisbrecher» unter den Studenten der verschiedenen Studienorte.

Was war für Dich persönlich das Highlight des Projektes?

Am Freitagnachmittag, nach dem Workshop und vor der nächsten Plenarveranstaltung, fuhren wir Studenten gemeinsam mit der Seilbahn ins Skigebiet hoch und genossen die Sonne ...
Würdest du die Teilnahme am Studentenprojekt Deinen Kolleginnen und Kollegen aus den kommenden Jahrgängen empfehlen? Ja, unbedingt! Es macht Spass, das erste Mal einen Kongress mit so breit gefächerter Thematik zu besuchen. Zudem lernt man meiner Meinung nach an einem hausärztlichen Kongress durchaus auch direkt Staatsexamen-relevante Inhalte.

\section{Der Tutor: Oliver Senn}

Als Tutor hatte ich Gelegenheit, das Aroser Studentenprojekt kennenzulernen. Im Rahmen von Kleingruppen (8-10 Studenten/ Studentinnen) haben mein Kollege und ich zwei Fallvignetten aus dem Praxisalltag kurz vorgestellt.

Nebst der Vermittlung von Fachwissen zu Diagnostik und Therapie galt es insbesondere, den Teilnehmerinnen und Teilnehmern die hausärztlichen Besonderheiten aufzuzeigen. Die spezifischen Vorkenntnisse aus der (langjährigen) Arzt-Patienten-Beziehung und die Berücksichtigung der Präferenzen der Patienten spielten in den Beispielen eine Schlüsselrolle bei der erfolgreichen Behandlung und Beratung. Die anschliessend sehr interaktive Falldiskussion verdeutlichte, dass es von den Teilnehmenden geschätzt wurde, die fachlichen Besonderheiten der medizinischen Grundversorgung zu thematisieren und damit auch Unterschiede zur Spitalversorgung besser zu verstehen.

\section{Ausblick}

Als neuer Projektverantwortlicher seit 2014, der die positive Stimmung bereits als Tutor kennengelernt hat, hat es mich gefreut, dass das Studentenprojekt dank der Unterstützung durch Mepha im Jahr 2014 von 24 auf 36 Teilnahmeplätze erweitert werden konnte, wobei die Anzahl Anmeldungen die Anzahl Plätze immer noch deutlich übertrifft, so dass weiterhin das Los über eine Teilnahme entscheiden muss.

Wir werden uns bemühen, in Zukunft auch junge Assistenzärzte und -ärztinnen im ersten und 2. Weiterbildungsjahr für eine Teilnahme rekrutieren zu können. Die Forschung hat klar gezeigt, dass die Weiterbildung eine entscheidende Phase für die spätere Berufswahl darstellt.

Das Projekt bietet die Chance, dass Assistenzärzte auf die Karrieremöglichkeiten in der Hausarztmedizin sensibilisiert werden und wertvolle Kontakte mit Tutoren und/oder zukünftigen Lehrpraktikern geknüpft werden können.

Ein spezieller Dank gilt den Tutoren und Referenten. Sie ermöglichen den Teilnehmern durch Ihr Engagement Einblicke in die Faszination der Hausarztmedizin und tragen wesentlich zum Erfolg des Projektes bei.

Korrespondenz:

PD Dr. med. Oliver Senn, Praxis MediS Zürich,

Institut für Hausarztmedizin Universität Zürich

Pestalozzistrasse 24, 8091 Zürich

oliver.senn[at]usz.ch 